\title{
A real-time artifact reduction algorithm based on precise threshold during short-separation optical probe insertion in neurosurgery
}

\author{
Weitao Li, Yangyang Liu, Yubing Liu, Yingxu Jiang and Zhiyu Qian* \\ Department of Biomedical Engineering \\ Nanjing University of Aeronautics and Astronautics \\ 29 Yudao Street, Nanjing 210016, P. R. China \\ *zhiyu@nuaa.edu.cn
}

Received 12 November 2015

Accepted 27 January 2016

Published 10 March 2016

\begin{abstract}
During neurosurgery, an optical probe has been used to guide the micro-electrode, which is punctured into the globus pallidus (GP) to create a lesion that can relieve the cardinal symptoms. Accurate target localization is the key factor to affect the treatment. However, considering the scattering nature of the tissue, the "look ahead distance (LAD)" of optical probe makes the boundary between the different tissues blurred and difficult to be distinguished, which is defined as artifact. Thus, it is highly desirable to reduce the artifact caused by LAD. In this paper, a real-time algorithm based on precise threshold was proposed to eliminate the artifact. The value of the threshold was determined by the maximum error of the measurement system during the calibration procession automatically. Then, the measured data was processed sequentially only based on the threshold and the former data. Moreover, $100 \mu \mathrm{m}$ double-fiber probe and two-layer and multi-layer phantom models were utilized to validate the precision of the algorithm. The error of the algorithm is one puncture step, which was proved in the theory and experiment. It was concluded that the present method could reduce the artifact caused by LAD and make the real boundary sharper and less blurred in real-time. It might be potentially used for the neurosurgery navigation.
\end{abstract}

Keywords: Look ahead distance; signal processing; Parkinson's disease; navigation; near-infrared spectrum.

\section{Introduction}

Parkinson's disease (PD) is a chronic and progressive disease involving the globus pallidus (GP), which is a gray matter mass surrounded by white matter deep within the brain. ${ }^{1,2}$ During neurosurgery, a probe is inserted into the GP to create a lesion that often relieves the cardinal symptoms of PD. ${ }^{3,4}$ Accurate target localization is the key factor

*Corresponding author.

This is an Open Access article published by World Scientific Publishing Company. It is distributed under the terms of the Creative Commons Attribution 4.0 (CC-BY) License. Further distribution of this work is permitted, provided the original work is properly cited. 
in the treatment. Thus, it is very important to obtain accurate anatomy information on the trajectory of the micro-electrode in real-time, especially determining the boundary between gray matter and white matter. Although magnetic resonance imaging (MRI) navigation is effective, it is costly and very complicated to operate. ${ }^{5-8} \mathrm{~A}$ safe, accurate and real-time navigation method in neurosurgery is still under exploration.

Near-infrared spectrum method plays a role in intraoperative navigation, which can obtain parameters of biological tissues real-time. ${ }^{9-12}$ The nearinfrared light $(650-950 \mathrm{~nm})$ penetrates more deeply up to several centimeters. Johns ${ }^{13}$ developed a portable, real-time spectrometer with fiber optic reflectance probe, which was utilized to assist neurosurgery. Giller ${ }^{14}$ introduced an intracranial probe with near-infrared light to identify gray-white matter junctions in stereotactic surgery. However, there was a slow change in the gray-white matter region, which blurred the boundary between white and gray matter. Here, we defined this slow change as the artifact.

The goal of this study is to eliminate the artifact. We explored this artifact caused by "look ahead distance" (LAD), which was introduced and discussed in some literatures. ${ }^{15,16}$ The spatial resolution of optical probe was related to the LAD. Due to LAD, the boundary between two tissues was not as sharp as that of MR images. Qian et al. ${ }^{17}$ proposed algorithms to determine LAD by both experimental and computational ways. Guo et al. ${ }^{18}$ introduced the approach of layer-based dispeller artifact of
LAD (LDA-LAD) by exponential function. However, it could not be implemented for the sequential measured data in real-time during the surgery and it was difficult to select the parameters of exponential function.

In this paper, we first provided the algorithm by using precision threshold to eliminate artifact and make the boundary clear to be distinguished. The measured data was processed sequentially (not the block data) by the present algorithm in real-time. In addition, the value of threshold was defined by the maximum error of the measurement system during each experiment. By using the algorithm and the threshold, the processed data could be divided into different tissues uniquely. The proposed method was validated by two-layer, multi-layer models and MR images in experiment. The maximum error was one puncture step, which could be also validated in the theory and experiment. The approach could make the precision of optical fiber probe insertion as much as MR images during neurosurgery.

\section{Materials and Methods}

\subsection{The artifacts caused by $L A D$}

The term of LAD means the "LAD" of the probe. ${ }^{17}$ It is the maximal length of the interrogated volume ahead of the probe (Fig. 1(a)), which depends on the optical properties of the tissues and the structure of the optical probe. LAD is defined by the reduced scattering coefficient $\left(\mu_{s}^{\prime}\right)$ or light intensity. Due to the LAD, the boundary between two tissues

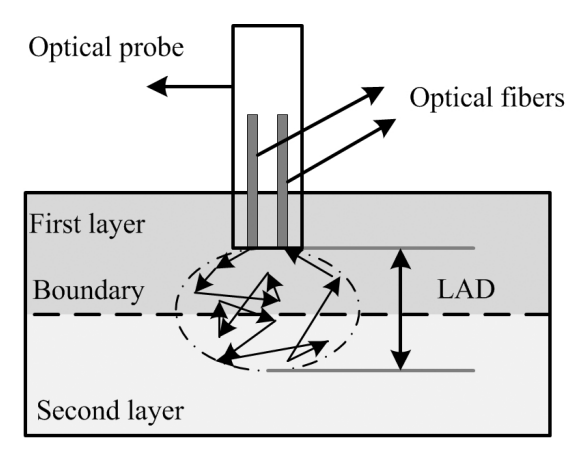

(a)

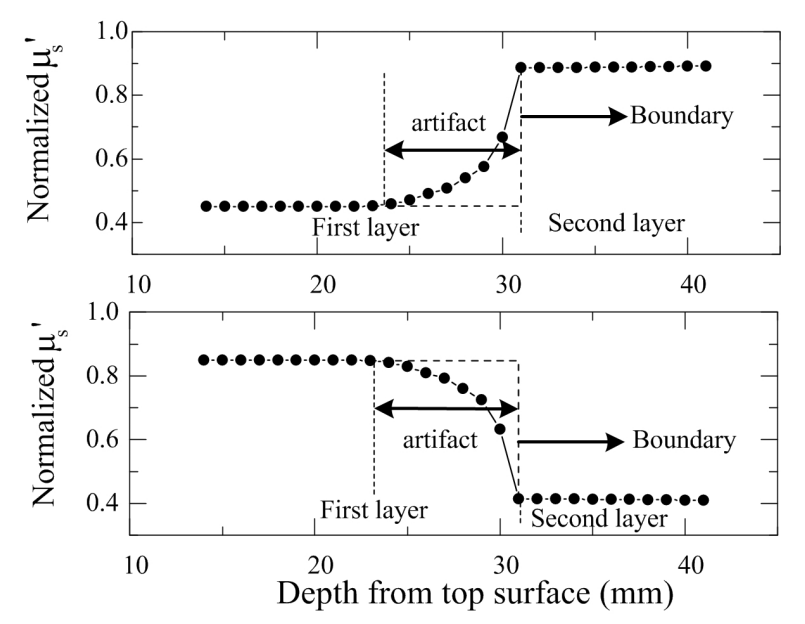

(b)

Fig. 1. Relationship between artifact and LAD. (a) Description of LAD (b) Definition of artifact by the curves of $\mu_{s}^{\prime}$. 
becomes less sharp and blurred, which is defined as artifact. In Fig. 1(b), the dash lines are the actual boundary between the two tissues. However, the artifact makes the boundary wider than the actual boundary. The purpose of this paper is to reduce the artifact and obtain the sharp boundary.

\subsection{The artifacts of $M R$ images of two-layer}

To compare the relationship between artifact and the actual boundary, MR images of two-layer models were investigated. The T1-weighted images were obtained by $1.5 \mathrm{~T}$ GE MRI machine. In Fig. 2(a), the puncture trajectory can be found in the phantom. The intensity of MR image along the trajectory is shown in Fig. 2(b). Based on the intensity, the boundary between two layers can be distinguished easily. MR images have a high spatial resolution compared with $\mu_{s}^{\prime}$ curves obtained by using optical fibers.

\subsection{Experimental setup}

The experimental system contained a continuous wave (CW) source and an intensity detector (Fig. 3). The source was tungsten-halogen light source with spectra range of 360-2000 nm (HL 2000, Ocean Optics Inc. Dunedin, Florida). The detector was a linear CCD spectrometer with wavelength ranging from 350 to $1100 \mathrm{~nm}$ (USB2000, Ocean Optics Inc. Dunedin, Florida).

The optical probe was composed of two fibers. One was connected with a source and the other was connected with a detector. The two fibers were $100 \mu \mathrm{m}$ in diameter and bundled into one stainless steel probe. The light was delivered into the tissue by one fiber and was collected by the other fiber (a)

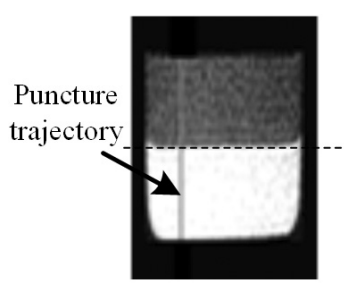

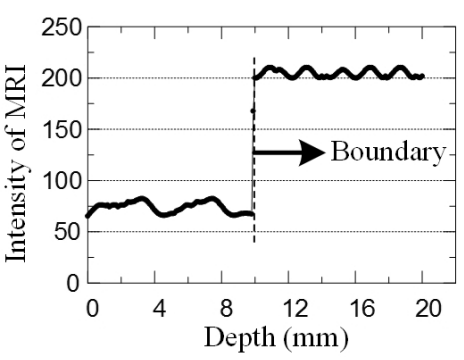

(b)
Fig. 2. Boundary of MR image. (a) The MR image of a twolayer model (b) the intensity of MR image along the trajectory.

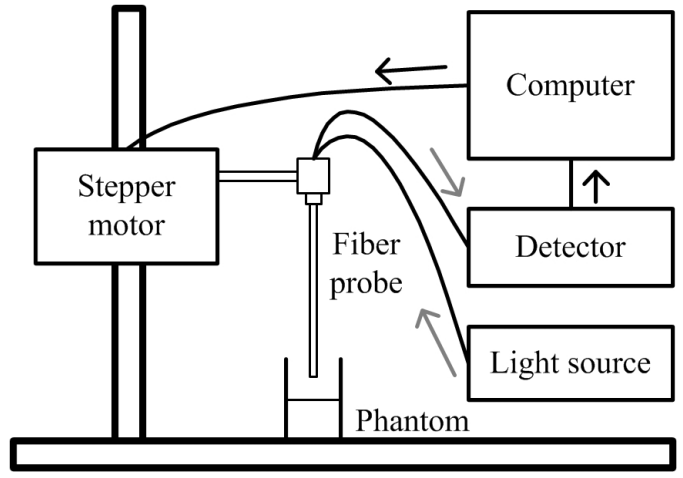

Fig. 3. The schematic representation of measurement system.

after scattering and absorption. In the experiment, the probe was inserted into the phantom step by step by a stepper motor. Here, the least puncture step size was $0.1 \mathrm{~mm}$. The integration time could be changed. The value of $\mu_{s}^{\prime}$ could be calculated from the spectrum. ${ }^{19,20}$

\subsection{The real-time artifact reduction algorithm}

The vital merit of the algorithm is that it processes the data sequentially. The incoming data must be evaluated rapidly for real-time considerations only based on the former data and itself. Compared with other algorithms processing the entire waveform (block data), this algorithm has better properties. When one raw data is obtained, it can be processed immediately.

The principle of the algorithm can be concluded by Eqs. (1)-(3).

$$
\begin{gathered}
Y(i)=\frac{1}{i} \sum_{j=1}^{i} X(j) \quad i=1,2, \ldots, N-1 \\
Y(i)=\frac{1}{N-1} \sum_{j=1}^{N-1} X(j) \quad i=N, N+1, \ldots, M-1 \\
Y(i)=X(i) \quad i=M,
\end{gathered}
$$

where $X(i)$ is the measured values of $\mu_{s}^{\prime},{ }^{19,20} Y(i)$ is the processed value from $X(i), i$ and $j$ are the index of the sampling point, the distance between two sampling points defines as one puncture step, $N$ and $M$ are the numbers of sample points in first layer outside artifact area and in the artifact area, respectively. The curve of $Y(i)$ can be utilized 


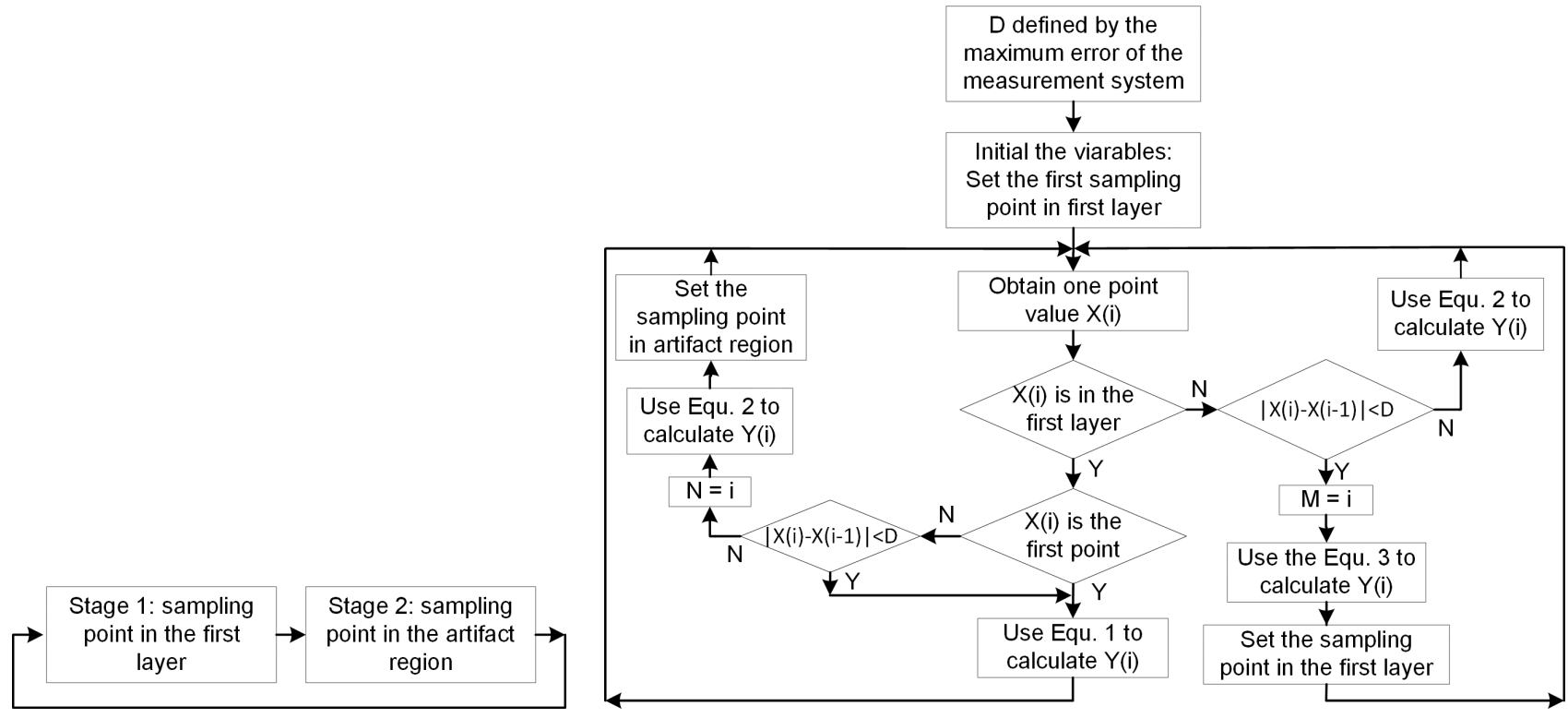

(a)

(b)

Fig. 4. Calculation steps of the proposed algorithm. (a) The whole process is divided into two-stage process model (b) block diagram of algorithm.

to identify the boundary of tissue easily and accurately.

The whole process is composed of several twostage processes, including sampling points in the first layer (stage 1) and in the artifact region (stage 2) shown in Fig. 4(a). If a sampling point enters the new layer from stage 2 , it will be sent to stage 1 in the algorithm. The algorithm can be carried out by the steps shown in Fig. 4(b) as follows.

Step 1: threshold setting

The threshold, defined as $D$, is not selected by subjective, which corresponded with the maximum error of the measurement system and the experiment conditions. We can place the probe into any standard calibration module and obtain the curve of $\mu_{s}^{\prime}$ or light intensity for $60 \mathrm{~s}$. Then, the maximum error of the data curve can be calculated, whose value is threshold.

\section{Step 2: data acquisition}

The measurement system obtained the value of $\mu_{s}^{\prime}$ in the position of optical probe, defined as $X(i)$. The $X(i)$ is influenced by the artifact, so it cannot be used to distinguish the actual boundary between two tissues and $X(i)$ must be processed.

Step 3: data processing

The $X(i)$ is processed and the $Y(i)$ can be obtained real-time. According to $Y(i)$, we can find which layer the tissue belongs to. In Fig. 4(a), the sampling point is divided into one of the two regions, including in the first layer outside the artifact region or in the artifact region. The artifact region is included in the first layer. Moreover, the number of point steps in the first and second layers, which are defined as $N$ and $M$, respectively.

At the beginning, the optical probe is in the first layer outside the artifact region.

(1) If $X(i)$ is the first sampling point, $Y(i)$ can be calculated by Eq. (1). Otherwise, if $\mid X(i)-$ $X(i-1) \mid<D \quad Y(i)$ can be calculated by Eq. (1); If $|X(i)-X(i-1)| \geq D, N$ is set to $i$, $Y(i)$ can be calculated by Eq. (2) and the sampling point is set in artifact region.

(2) Here, the optical probe is in the artifact region. If $|X(i)-X(i-1)| \geq D, Y(i)$ can be calculated by Eq. (2). If $|X(i)-X(i-1)|<D$, the value of $M$ is set to $i, Y(i)$ is calculated by Eq. (3) and the sampling point is set in the second layer.

(3) To simplify the algorithm, we reset the second layer to first layer. Then, the algorithm returns to step 1).

In the present algorithm, the measured data is processed by the algorithm sequentially. So, the maximum spatial error is one puncture step between two sampling points. For example, if a new coming point is in the second layer, it might be 
considered as the point in the first layer. However, the next points will be in the correct layer.

\subsection{Preparation of phantom model}

The phantom models consisted of a mixture of Intralipid and gelatin in proportions to simulate the optical properties of brain tissues. The actual $\mu_{s}^{\prime}$ values and thickness of different layers were measured by a dual-channel and frequency-domain Oximeter (ISS, Champaign, IL).

The two-layer phantom models were constructed to simulate a top layer (or white matter layer) overlying a bottom layer (or gray matter layer). The thickness of each layer was $10 \mathrm{~mm}$. The nearinfrared measurements were done every $0.1 \mathrm{~mm}$ or $0.2 \mathrm{~mm}$ as the optical probe was puncturing from the top layer to the bottom layer (Fig. 5(a)). To explore the artifact caused by LAD in different tissues, the two-layer phantom models were divided into base-up and base-down groups. In base-up model, the value of $\mu_{s}^{\prime}$ in the first layer is smaller than that in the second layer. However, in basedown model, the value of $\mu_{s}^{\prime}$ in the first layer is bigger than that in the second layer. Each model was divided into four different groups. The values of $\mu_{s}^{\prime}$ in each group, measured by Oximeter ISS, are listed in Table 1 (base-up groups) and Table 2 (base-down groups). Then, the measured data, obtained from model Up_4 and Dn_4, were processed by the present algorithm. The data measurement began at $5 \mathrm{~mm}$ above the boundary of the top and middle layers. The effect of algorithm would be proved by this experiment.

Moreover, the three-layer models were defined as top, middle and bottom layer, respectively. Compared with LAD, the thickness of the layer was important factor to affect availability of algorithm.
Table 1. $\quad \mu_{s}^{\prime}$ of base-up models $\left(\mathrm{cm}^{-1}\right)$

\begin{tabular}{lcrrr}
\hline & Up_1 & Up_2 & Up_3 & Up_4 \\
\hline Top $\mu_{s}^{\prime}$ & 6.83 & 7.1 & 7.1 & 7.3 \\
Bottom $\mu_{s}^{\prime}$ & 12.1 & 16.4 & 18.3 & 20.4 \\
\hline
\end{tabular}

Table 2. $\mu_{s}^{\prime}$ of base-down models $\left(\mathrm{cm}^{-1}\right)$.

\begin{tabular}{lrrrr}
\hline & Dn_1 & Dn_2 & Dn_3 & Dn_4 \\
\hline Top $\mu_{s}^{\prime}$ & 12.7 & 16.6 & 18.4 & 21.4 \\
Bottom $\mu_{s}^{\prime}$ & 6.9 & 6.9 & 7.1 & 7.2 \\
\hline
\end{tabular}

When the thickness of layer was smaller than LAD, the optical fiber could not distinguish it or "see" it. According to the thickness of middle layer, the models could be divided into two groups. In group $\mathrm{L}$, the thickness of middle layer was bigger than LAD. In group $\mathrm{S}$, the thickness equaled to LAD. According to the value of $\mu_{s}^{\prime}$, group $\mathrm{L}$ and group $\mathrm{S}$ were divided into four models respectively, including base-down-base (bdb), base-up-base (bub), base-down-down (bdd) and base-up-up (buu) models. The parameters of two groups, measured by Oximeter ISS, are listed in Table 3. The puncture step was $0.1 \mathrm{~mm}$. The actual thickness of each layer was obtained by the $\mu_{s}^{\prime}$ curves.

During neurosurgery navigation, the measured data was obtained from the multi-layer models. Therefore, we developed two groups of multi-layer models to validate the precision of the present algorithm. Every multi-layer model has seven layers. Group L was composed of Lbdb, Lbub, Lbdd and Lbuu models. From top to bottom the actual thickness of each phantom layer was $3,6,2.8,3,3.1$, 3 and $2.1 \mathrm{~mm}$, which were bigger than LAD. Group $\mathrm{S}$ was composed of Sbdb, Sbub, Sdd and Sbuu models. The actual thickness of each phantom layer

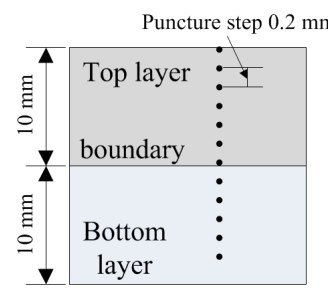

(a)

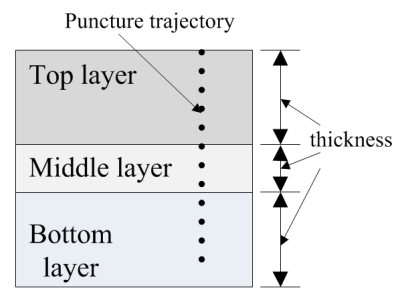

(b)

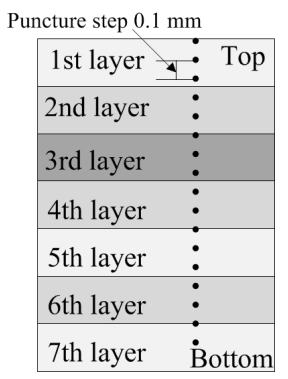

(c)

Fig. 5. The schemes of the phantom and experiments. (a) Two-layer model (b) three-layer model (c) multi-layer model. 
Table 3. $\mu_{s}^{\prime}$ and the thickness of three-layer models.

\begin{tabular}{|c|c|c|c|c|c|c|c|c|c|c|c|c|c|c|c|c|}
\hline \multirow[b]{2}{*}{ Layer } & \multicolumn{2}{|c|}{ Lbdb } & \multicolumn{2}{|c|}{ Lbub } & \multicolumn{2}{|c|}{ Lbdd } & \multicolumn{2}{|c|}{ Lbuu } & \multicolumn{2}{|c|}{ Sbdb } & \multicolumn{2}{|c|}{ Sbub } & \multicolumn{2}{|c|}{ Sbdd } & \multicolumn{2}{|c|}{ Sbuu } \\
\hline & $\mu_{s}^{\prime}$ & $\mathrm{T}$ & $\mu_{s}^{\prime}$ & $\mathrm{T}$ & $\mu_{s}^{\prime}$ & $\mathrm{T}$ & $\mu_{s}^{\prime}$ & $\mathrm{T}$ & $\mu_{s}^{\prime}$ & $\mathrm{T}$ & $\mu_{s}^{\prime}$ & $\mathrm{T}$ & $\mu_{s}^{\prime}$ & $\mathrm{T}$ & $\mu_{s}^{\prime}$ & $\mathrm{T}$ \\
\hline Top & 15 & 3 & 7.3 & 3 & 25 & 3 & 7.3 & 3 & 15 & 3 & 7.3 & 3 & 25 & 3 & 7.3 & 3 \\
\hline Middle & 7.3 & 3 & 17.3 & 3 & 17.3 & 3 & 17.3 & 3 & 7.3 & 1.1 & 17.3 & 1.3 & 17.3 & 1.3 & 17.3 & 0.9 \\
\hline Bottom & 15 & - & 7.3 & - & 7.3 & - & 25 & - & 15 & - & 7.3 & - & 7.3 & - & 25 & - \\
\hline
\end{tabular}

Note: The units of $\mu_{s}^{\prime}$ is $\mathrm{cm}^{-1}$; T means the thickness of layer, the units of which is mm; $\mathrm{L}^{* * *}$ means the thickness of middle layer is larger than LAD; $\mathrm{S}^{* * *}$ means the thickness of middle layer is smaller than LAD.

was $3,0.9,1.1,1.1,1.2,1.3$ and $1.4 \mathrm{~mm}$ from top to bottom. The thickness of the layers (from second layer and sixth layer) was smaller than LAD. The puncture step is $0.1 \mathrm{~mm}$.

\section{Results}

\subsection{Artifacts and two-layer models}

The artifacts defined by the curves of $\mu_{s}^{\prime}$ in base-up and base-down groups are shown in Fig. 6. Then,

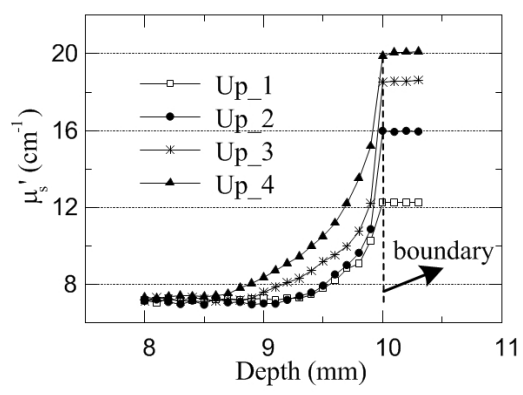

(a)

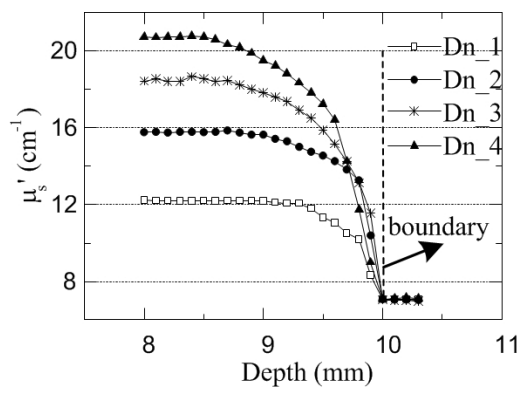

(b)

Fig. 6. Artifacts in phantom models (a) base-up modes (b) base-down models. another actual two-layer phantom models were measured by the measurement system with optical probes. The optical parameters of two models were the same as group Up_4 and group Dn_4 in Tables 1 and 2, respectively. The measurement started at $5 \mathrm{~mm}$ above the boundary between two layers. That was the real boundary at $5 \mathrm{~mm}$. The measured data are shown in Figs. 7(a) and 7 (c). The artifacts made the boundary between two layers unclear. Then, the processed data were obtained by using the present algorithm. The artifacts were reduced and the boundary can be found easily and precisely by the curves in Figs. 7 (b) and $7(\mathrm{~d})$, which are at $5.2 \mathrm{~mm}$. Therefore, the error of the algorithm was $0.2 \mathrm{~mm}$, which equaled to one puncture step.

\section{2. $\quad$ Three-layer models}

The data curves in group $\mathrm{L}$ and group $\mathrm{S}$ are shown in Figs. 8 and 9, respectively. The dot lines indicate the measured data and the solid lines indicate the processed data. From the actual thickness in Table 3, the real boundaries are at 3 and $6 \mathrm{~mm}$. In group $\mathrm{S}$, all the real first boundaries are at $3 \mathrm{~mm}$. The real second boundaries are at 4.1, 4.3, 4.3 and $3.9 \mathrm{~mm}$ for Sbdb, Sbub, Sbdd and Sbuu models, respectively. The boundaries, calculated by the present algorithm, are shown in Table 4 . The errors are $0.1 \mathrm{~mm}$, which is equal to one puncture step.

\subsection{Multi-layer models}

In the multi-layer models, the measured data and processed data are shown in Figs. 8 and 9 . 


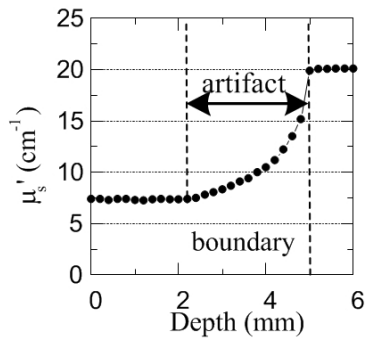

(a)

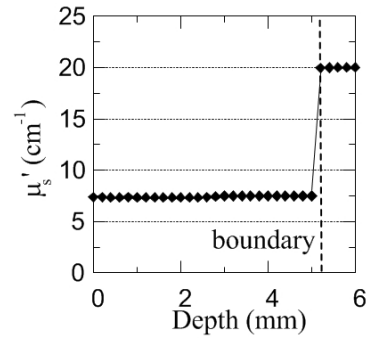

(b)

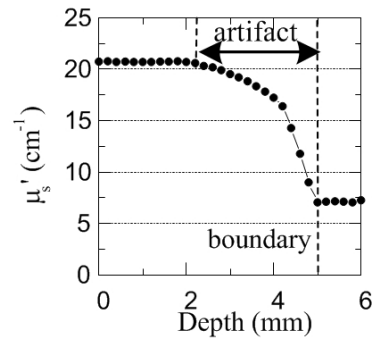

(c)

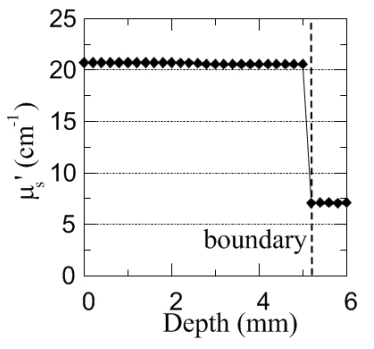

(d)

Fig. 7. The $\mu_{s}^{\prime}$ curves of two-layer models (a) the measured data of Up_4 model (b) the processed data of Up_4 model (c) the measured data of Dn_4 model (d) the processed data of Dn_4 model.

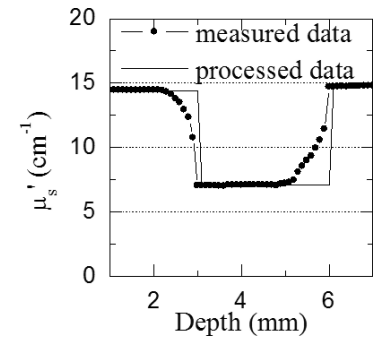

(a)

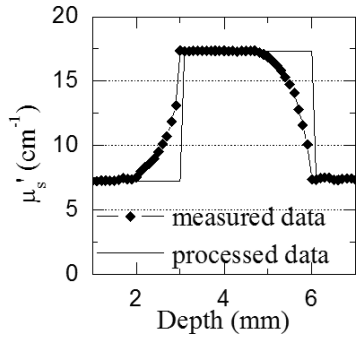

(b)

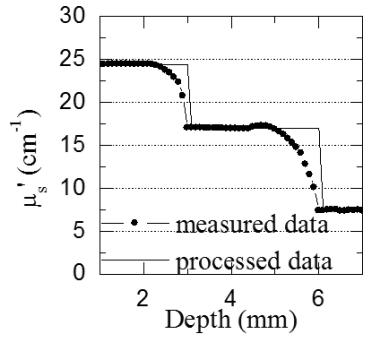

(c)

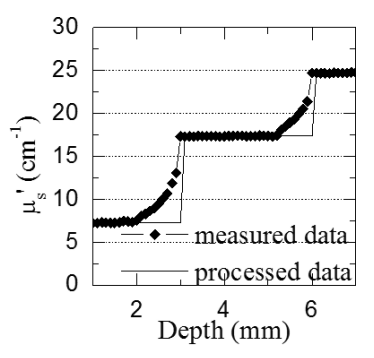

(d)

Fig. 8. The $\mu_{s}^{\prime}$ curves of three-layer group L (a) Lbdb model (b) Lbub model (c) Lbdd model (d) Lbuu model.

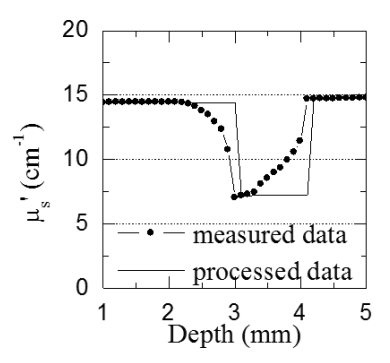

(a)

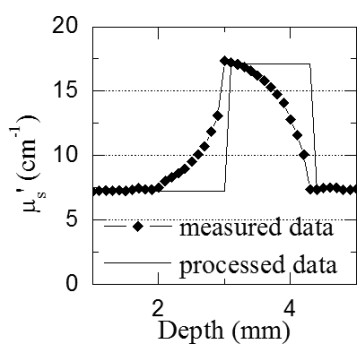

(b)

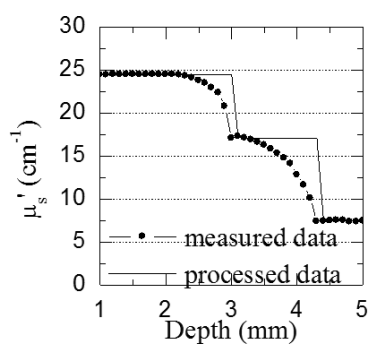

(c)

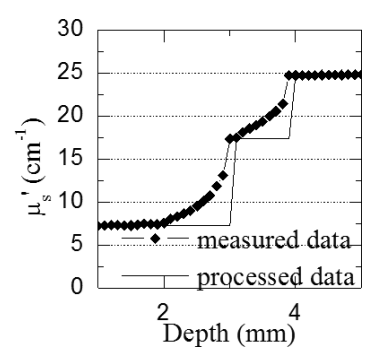

(d)

Fig. 9. The $\mu_{s}^{\prime}$ curves of three-layer group S (a) Sbdb model (b) Sbub model (c) Sbdd model (d) Sbuu model.

Table 4. The boundaries calculated by present algorithm (mm).

\begin{tabular}{lcccccccc}
\hline & Lbdb & Lbub & Lbdd & Lbuu & Sbdb & Sbub & Sbdd & Sbuu \\
\hline 1st boundary & 3.1 & 3.1 & 3.1 & 3.1 & 3.1 & 3.1 & 3.1 & 3.1 \\
2nd boundary & 6.1 & 6.1 & 6.1 & 6.1 & 4.2 & 4.4 & 4.4 & 4.0 \\
\hline
\end{tabular}

Due to the value of $\mathrm{LAD}$, the multi-layer models were divided into two groups, including group LM (Fig. 10(a)) and group SM (Fig. 10(b)). In Fig. 10(a), the solid lines are the processed data, which indicate the positions of boundaries are 3.1,
6.1, 8.9, 11.9, 14.9 and $18 \mathrm{~mm}$. In Fig. 10(b), the solid lines indicate the positions of boundaries are $3.1 \mathrm{~mm}, 4 \mathrm{~mm}, 5.1 \mathrm{~mm}, 6.2 \mathrm{~mm}, 7.4 \mathrm{~mm}$ and $8.7 \mathrm{~mm}$. Compared with the actual boundaries, the errors are $0.1 \mathrm{~mm}$, which is equal to one puncture step. 


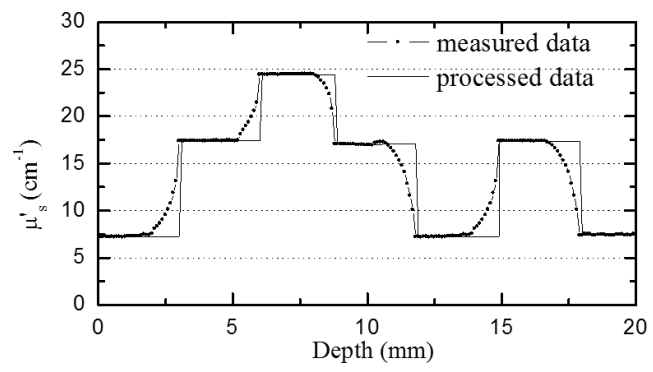

(a)

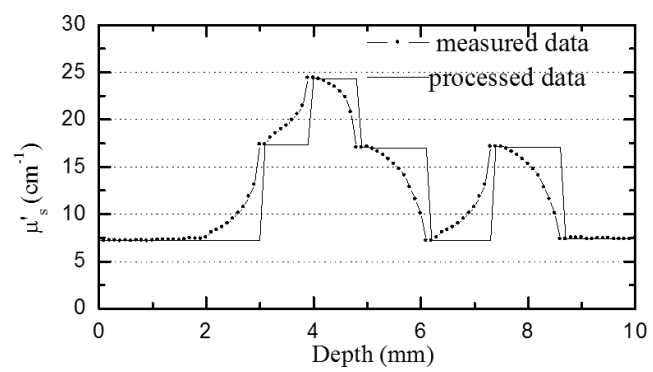

(b)

Fig. 10. The $\mu_{s}^{\prime}$ curves of multi-layer models (a) the thickness of middle layer is larger than LAD (b) the thickness of middle layer is smaller than LAD.

\section{Discussion}

Nowadays, near-infrared minimally invasive technique is effectively utilized to distinguish gray matter, white matter, and CSF to identify many subcortical targets of interest. ${ }^{21,22}$ In this paper, we present an algorithm by using precision threshold to reduce the artifact caused by LAD in real-time. A $100 \mu \mathrm{m}$ double-fiber probe system and two-layer and multi-layer phantom models were utilized to verify the present algorithm. In Fig. 6, compared with the actual boundary (dash line), the artifacts were different in each model, though the same optical probe and the system were utilized in the experiment. Furthermore, the artifacts depended on not only the parameters of optical fibers, but also the value of $\mu_{s}^{\prime}$ of two layers. Therefore, the artifacts made the boundary of two layers blurred and unintelligible. For example, in Figs. 7(a) and 7(c), the boundaries are not clearly in the curves of measured data. Therefore, it was important to find a new method to reduce artifact. Furthermore, the boundary could be found easily and precisely by the processed data curves in Figs. 7(b) and 7(d). The most advantage of this method was real-time. Compared with the MR images, the spatial resolution of the reduced data was similar as MR images. Furthermore, this method was low-cost.

The needle-like optical probe would be used in the navigation of neurosurgery in human brain, which was considered as the multi-layer model. ${ }^{23,24}$ Therefore, several multi-layer models were investigated. The thickness of middle layer was the most important factor in three-layer models' experiments. Based on it, the models were divided into two groups. The results from all the multi-layer models experiments proved that the minimum layer-resolution of the optical fiber depended on the value of $\mathrm{LAD}$.

In the present algorithm, the value of threshold was an important factor to affect the precision. A lot of research gave approaches to obtain the threshold value by subjective or human-computer interaction. ${ }^{25}$ In this paper, the threshold was objectively defined according to the maximum error of the measurement system in each experiment. It did not depend on the value of optical parameter or the difference of tissues. Therefore, the maximum error could be acquired during the calibration process. If the $\mu_{s}^{\prime}$ difference was smaller than the maximum error, the difference could not be distinguished by the instrument. The definition method was reasonable. The maximum error was related to the instrument measurement conditions, including the background light and the optical parameter of tissue.

According to the theory and experiment analysis, the precision of the algorithm was one puncture step compared with the thickness defined by measured data, which does not depend on the thickness of each layer. Here, the algorithm system is the causal system. The current data is only dependent on the former data. In the algorithm implemented by computer, the computing time is nearly negligible. Furthermore, the algorithm was not influenced by the different LAD. The LAD is related to the shape and size of the optical probes. It also depends on the tissue's optical parameter and the difference of two tissues. So it is hard to distinguish the boundary of the two tissues by LAD automatically. In this paper, the algorithm has strong robust stability to the different LAD.

\section{Conclusion}

A real-time artifact reduction algorithm based on precision threshold was proposed in this paper. The 
reduction algorithm could decrease the artifact caused by LAD. The processed curves will be utilized to distinguish the boundary between two tissues. The precision of the present algorithm equals one puncture step in the experiment. This algorithm could make the navigation by near-infrared light more accurately.

\section{Acknowledgment}

All the work was completed in Biomedical Photonics Laboratory of NUAA (Nanjing University of Aeronautics and Astronautics). The work was supported by "the Fundamental Research Funds for the Central Universities", No. NS2015032.

\section{References}

1. L. Zhao, L. V.-Metman, J. H. Kim, C. C. Liu, F. A. Lenz, "EMG activity and neuronal activity in the internal globus pallidus (GPi) and their interaction are different between hemiballismus and apomorphine induced dyskinesias of Parkinson's disease (AID)," Brain Res. 1603, 50-64 (2015).

2. C. R. Johannes, K. Y. Michele, C. Kim, P. Luo, J. M. William, M. W. Frances, S. Matthew, F. Kenneth, R. Domenic, "Neuropsychological changes following deep brain stimulation surgery for Parkinson's disease: Comparisons of treatment at pallidal and subthalamic targets versus best medical therapy," $J$. Neurol. Neurosurg. Psychiatry 86, 622-629 (2015).

3. J. Z. Andrew, J. N. Gerald, D. Michel, S. T. Robert, "Movement-related discharge in the macaque globus pallidus during high-frequency stimulation of the subthalamic nucleus," J. Neurosci. 35, 3978-3989 (2015).

4. A. B. David, M. D. Anders, A. F. Maria, "Deepbrain stimulation of the subthalamic nucleus or the pars interna of the globus pallidus in Parkinson's disease," The New Eng. J. Med. 345, 956-963 (2001).

5. J. A. Obeso, C. M. Olanow, M. C. Rodriguez-Oroz, P. Krack, R. Kumar, A. E. Lang, "Diffuse optical imaging of brain activation: Approaches to optimizing image sensitivity, resolution," NeuroImage 23, 275-288 (2004).

6. H. Lai, R. Y. John, L. A. Daniel, J. K. Yu-Chieh, I. S. Yen-Yu, "Functional MRI reveals frequencydependent responses during deep brain stimulation at the subthalamic nucleus or internal globus pallidus," NeuroImage 84, 11-18 (2014).

7. K. Joshua, U. Maren, M. Rosalyn, F. Guillaume, M. Andre, M. Laura, W. Mark, T. John, Y. Tarek, Z.
Ludvic, H. Marwan, L. Patricia, F. Karl, F. Tom, "Resting state functional MRI in Parkinson's disease: The impact of deep brain stimulation on 'effective' connectivity," Brain 173, 1130-1144 (2014).

8. J. A. Frederick, R. B. Bastiaan, M. Philipp, S. Klaus, G. Bozena, "Update on diffusion MRI in Parkinson's disease and atypical parkinsonism," $J$. Neurol. Sci. 332, 21-29 (2014).

9. F. Jobsis, "Non-invasive infrared monitoring of cerebral and myocardial oxygen sufficiency and circulatory Parameters," Science 198, 1264-1267 (1977).

10. L. Dai, Z. Qian, K. Li, T. Yang, H. Wang, "In vivo detection of reduced scattering coefficient of $\mathrm{C} 6$ glioma in rat brain tissue by near-infrared spectroscopy," J. Biomed. Opt. 13, 044003 (2008).

11. S.-H. Kong, Y.-W. Noh, Y.-S. Suh, H. S. Park, H.-J. Lee, K. W. Kang, H. C. Kim, Y. T. Lim, H.-K. Yang, "Evaluation of the novel near-infrared fluorescence tracers pullulan polymer nanogel and indocyanine green $/ \gamma$-glutamic acid complex for sentinel lymph node navigation surgery in large animal models," Gastric Cancer 18, 55-64 (2015).

12. N. R.-Labarbe, A. Fenoglio, A. Aggarwal, M. Dehaes, St. A Carp, M. A. Franceschini, P. E. Grant, "Near-infrared spectroscopy assessment of cerebral oxygen metabolism in the developing premature brain," J. Cerebral Blood Flow Metabol. 32, 481-488 (2012).

13. J. Maureen, G. Cole, H. Liu, "Computational and in vivo investigation of optical reflectance from human brain to assist neurosurgery," J. Biomed. Opt. 3, 437-445 (1998).

14. A. G. Cole, J. Maureen, H. Liu, "Use of an intracranial near-infrared probe for localization during stereotactic surgery for movement disorders," $J$. Neurosurg. 93, 498-505 (2000).

15. A. G. Cole, H. Liu, G. Prem, V. Sundar, Y. Umar, C. G. Dwight, "Validation of a near-infrared probe for detection of thin intracranial white matter structures," J. Neurosurg. 98, 1299-1306 (2003).

16. J. R. Mourant, T. M. Johnson, G. Los, I. J. Bigio, "Non-invasive measurement of chemotherapy drug concentrations in tissue: Preliminary demonstrations of in vivo measurement," Phys. Med. Biol. 44, 1397-1417 (1999).

17. Z. Qian, S. V. Sundar, Y. Gu, A. G. Cole, H. Liu, Look-Ahead Distance" of a fiber probe used to assist neurosurgery: Phantom and Monte Carlo study," Opt. Express 11, 1844-1855 (2003).

18. L. Guo, Z. Qian, W. Li, "Research on look ahead distance (LAD) in the mini-invasive penetration track of biology tissue by near infrared spectroscopy (NIRS) technique," Annual Meeting of the Chinese Optical Society, Quanzhou, Fujian (2008). 
19. J. Maureen, H. Liu, "Calculating the reduced scattering coefficient of turbid media from a single optical reflectance signal," Proc. SPIE 4958, 251-258 (2003).

20. J. Maureen, A. G. Cole, C. G. Dwight, H. Liu, "Determination of reduced scattering coefficient of biological tissue from a needle-like probe," Opt. Express 13, 4828-4842 (2005).

21. L. J. Elizabeth, J. Huang, R. A. Nadeem, J. G. Ginger, L. B. Carol, S. Yukio, R. B. Richard, A. L. Douglas, M. L. Mario, "Detection of sentinel lymph nodes in minimally invasive surgery using indocyanine green and near-infrared fluorescence imaging for uterine and cervical malignancies," Gynecol. Oncol. 133, 274-277 (2014).

22. M. Ikeda, A. Matsushita, "Matsushita Reflectance of rat brain structures mapped by an optical fiber technique," J. Neurosci. Methods 2, 9-17 (1980).
23. M. Dehaes, P. E. Grant, D. D. Sliva, N. R.-Labarbe, R. Pienaar, D. A. Boas, M. A. Franceschini, J. Selb, "Assessment of the frequency-domain multidistance method to evaluate the brain optical properties: Monte Carlo simulations from neonate to adult," Biomed. Opt. Express 2, 552-567 (2011).

24. R. S. Agnes, A.-M. Broome, J. Wang, A. Verma, K. Lavik, J. P. Basilion, "An optical probe for noninvasive molecular imaging of orthotopic brain tumors overexpressing epidermal growth factor receptor," Mol. Cancer Ther. 11, 2202-2211 (2012).

25. J. R. Lee, M. T. Madsen, D. Bushnel, Y. Menda, "A threshold method to improve standardized uptake value reproducibility," Nucl. Med. Commun. 21, 685-690 (2000). 